\title{
Relationship Between Sarcopenia and Both Physical Activity and Lifestyle in Patients With Chronic Liver Disease
}

\author{
Kazuki Ohashi ${ }^{\mathrm{a}, \mathrm{b}}$, Toru Ishikawa ${ }^{\mathrm{c}}$, Asami Hoshi ${ }^{\mathrm{d}}$, Mitsuyuki Suzukie, Yuta Mitobe ${ }^{\mathrm{f}}$, \\ Etsuko Yamada ${ }^{a}$, Hansani Madushika Abeywickramaa ${ }^{a}$ Nao Sekia, \\ Chikayo Koyama ${ }^{\text {a }}$, Hagiko Aokig, Yu Koyama, ${ }^{\text {a }}$,
}

\begin{abstract}
Background: Sarcopenia can affect the prognosis of patients with cirrhosis or hepatocellular carcinoma. Exercise therapy and nutritional therapy are carried out to prevent processing sarcopenia. In addition, changing lifestyle is also important. However, there are only few reports on the physical activities (PAs) and lifestyle of chronic liver disease patients and their association with sarcopenia. The aim of this study is to examine the relationship between sarcopenia in patients with chronic liver disease and both PA and lifestyle.

Methods: A total of 214 out-patients with chronic liver disease were enrolled into the present study. All patients were evaluated for with or without sarcopenia based on the sarcopenia diagnostic criteria of the Japan Society of Hepatology. Then, patient's characteristics and laboratory parameters were divided into two groups with or without sarcopenia and compared. In continuous variable with significant difference in univariate analysis, cut-off value was calculated by receiver operating characteristic curve. We determined which factors were associated with sarcopenia in univariate analyses, and variables significant in the univariate analyses were entered in a multivariable logistic regression model.
\end{abstract}

Results: Patients with chronic liver disease had a prevalence of sarcopenia of $12.6 \%$ in this study. Sarcopenia patients were older $(76.48$ \pm 6.69 versus $66.97 \pm 11.19$ years old; $\mathrm{P}<0.01)$, had lower body

Manuscript submitted September 28, 2018, accepted October 12, 2018

aDepartment of Nursing, Niigata University Graduate School of Health Sciences, Niigata, Japan

${ }^{b}$ Department of Nursing, Saiseikai Niigata Daini Hospital, Niigata, Japan

cDepartment of Gastroenterology and Hepatology, Saiseikai Niigata Daini Hospital, Niigata, Japan

dDepartment of Medical Radiology, Saiseikai Niigata Daini Hospital, Niigata, Japan

eDepartment of Pharmacology, Saiseikai Niigata Daini Hospital, Niigata, Japan

fDepartment of Nursing, Teikyo University, Faculty of Medical Technology, Tokyo, Japan

gDepartment of Nursing, Chukyo Gakuin University, Gifu, Japan

${ }^{\text {h}}$ Corresponding Author: Yu Koyama, Department of Nursing, Niigata University Graduate School of Health Sciences, 2-746 Asahimachi, Niigata 951-

8518, Japan. Email: yukmy@clg.niigata-u.ac.jp

doi: https://doi.org/10.14740/jocmr3640 mass index (BMI) $\left(20.84 \pm 2.44\right.$ versus $\left.23.76 \pm 3.72 \mathrm{~kg} / \mathrm{m}^{2} ; \mathrm{P}<0.01\right)$, lower PA (6.6 (2.34 - 19.90) versus 16.5 (6.60 - 41.23) metabolic equivalents (METs)-h/week; $\mathrm{P}<0.01)$ and longer total time sitting and lying on the day $(7.43 \pm 4.09$ versus $5.68 \pm 3.17 \mathrm{~h} /$ day; $\mathrm{P}=0.01)$; retirement status $(81.5 \%$ versus $48.1 \% ; \mathrm{P}<0.01)$ and low frequency of driving ( $40 \%$ versus $20 \%$; $\mathrm{P}=0.01$ ) were higher in sarcopenia patients than in non-sarcopenia patients. The independent predictive factors of sarcopenia, analyzed with logistic regression, were age (odds ratio (OR): 5.89, 95\% confidence interval (CI): 2.15 - 16.20; P $<0.01$ ), BMI (OR: 4.77, 95\% CI: $1.87-12.10$; $\mathrm{P}<0.01)$ and PA (OR: $3.65,95 \%$ CI: 2.15 - 16.20; P<0.01).

Conclusion: Sarcopenia patients' lifestyle characteristics were longer sedentary time and low frequency of driving, high retirement. Independent predictive factors of sarcopenia were elderly, low BMI and low PA. For these patients, intervention in the lifestyle for prevention of sarcopenia may be effective for patients with chronic liver disease.

Keywords: Sarcopenia; Physical activity; International Physical Activity Questionnaire; Chronic liver disease

\section{Introduction}

In 1989, the term "sarcopenia" was coined to describe a decrease in muscle mass caused by aging [1]. It was later refined by the European Working Group on Sarcopenia in Older People (EWGSOP) to define a syndrome characterized by massive loss of progressive and systemic skeletal muscle with consequences such as physical disorder and decreased quality of life [2]. Similar muscle loss not associated with aging was defined as secondary sarcopenia $[2,3]$. In general, sarcopenia can lead to reduced physical capability, poorer quality of life, unfavorable metabolic effects, disability and mortality in elderly patients [4]. In turn, studies have shown a strong relationship between sarcopenia and physical inactivity $[5,6]$, and in elderly sufferers, moderate resistance training might prevent sarcopenia and increase skeletal muscle mass [7].

Patients with chronic liver disease can show decreased skeletal muscle mass in the earlier stages of disease due to impaired protein synthesis [8]. Consequently, a high proportion of these patients develop sarcopenia regardless of age [9-13], 
and the presence of sarcopenia is reported to worsen the prognosis of patients with hepatocellular carcinoma and cirrhosis $[9,10,14,15]$. In Japan, $10-70 \%$ of patients with liver cirrhosis or hepatocellular carcinoma are also diagnosed with sarcopenia, prompting the Japan Society of Hepatology to establish guidelines for the diagnosis and treatment of sarcopenia in liver disease (first edition) in 2016 [16]. Previous studies indicated that exercise and nutritional therapy improve physical function and increase muscle mass in patients with chronic liver disease $[17,18]$; however, patients with non-alcoholic fatty liver disease and cirrhosis wait-listed for liver transplantation tend to have low levels of physical activity (PA) compared to healthy adults $[19,20]$. In addition, patients with severe liver cirrhosis and esophageal varices are limited in their ability to exercise [21, 22].

It would therefore seem that appropriate intervention according to individual PA and lifestyle is important for the treatment and prevention of sarcopenia; however, few studies on such factors and their association with sarcopenia are available for patients with chronic liver disease. This study thus aimed to examine how sarcopenia in patients with chronic liver disease is related to PA and lifestyle.

\section{Materials and Methods}

\section{Study population and design}

This was a cross-sectional study conducted at the Chronic Liver Disease Outpatient Clinics, Saiseikai Niigata Daini Hospital, Niigata, Japan. Patients who received computed tomography (CT) for screening hepatocellular carcinoma (HCC) at Saiseikai Niigata Daini Hospital, were enrolled into the present study. Ethics approval was obtained from the ethics committee of Niigata University (approval number: 2017-0082) and Saiseikai Niigata Daini Hospital (approval number: E1706). Participants signed an informed consent before being enrolled in the study. Written, informed consent for participation was obtained from each patient on attendance at the outpatient clinic prior to enrollment in the study. Patients were excluded on the following criteria: unable to measure hand grip strength, no response to questionnaire and dementia.

\section{Patient characteristics and laboratory parameters}

Patient characteristics were obtained from questionnaires and medical records, namely, age, sex, body mass index (BMI), etiology of liver disease, HCC, drug therapy, living alone or living together, smoking, drinking, frequency of driving a motor vehicle, employment status, home task, opinions concerning diet and exercise.

Blood samples were obtained from each patient before CT for biochemical and hematological evaluation to measure the following levels: serum albumin (Alb), alkaline phosphatase (ALP), alanine transaminase (ALT), aspartate aminotransferase (AST), blood urea nitrogen (BUN), creatine kinase $(\mathrm{CK})$, creatinine (Cre), cholinesterase (ChE), estimated glo- merular filtration rate (eGFR), hemoglobin ( $\mathrm{Hb})$, hemogobinA1c (HbA1c), $\alpha$-fetoprotein (AFP), potassium (K), sodium $(\mathrm{Na})$, prothrombin time-international normalized ratio (PTINR), total bilirubin (T-bil), total cholesterol (T-cho), total protein (TP), triglyceride (TG), red blood cell count (RBC), white blood cell count (WBC), total lymphocyte count (TLC), platelet count (Plt) and controlling nutritional status index score (CONUT-score). Malnutrition was scored based on serum Alb, T-cho and TLC [23]. The Fib4-index, which evaluates liver fibrosis, was scored based on age, ALT, AST and Plt [24-26]. The albumin-bilirubin grade (ALBI-grade) was proposed as a simple and objective assessment of liver function, in which the score is based solely on the serum albumin and bilirubin levels [27]. HbA1c was expressed as a National Glycohemoglobin Standardization Program (NGCP) unit.

\section{Sarcopenia assessments}

This study defined sarcopenia according to recommendations by the Japan Society of Hepatology [16], as skeletal mass index (SMI) plus muscle strength, with respective cut-off values for SMI of $42 \mathrm{~cm}^{2} / \mathrm{m}^{2}$ for male and $38 \mathrm{~cm}^{2} / \mathrm{m}^{2}$ for female based on CT, and for handgrip strength of $<26 \mathrm{~kg}$ for male and $<18 \mathrm{~kg}$ for female. Each patient's SMI was calculated using CT images, with the cross-sectional area of skeletal muscle measured at the third lumbar vertebra (L3) level. Calculated cross-sectional areas $\left(\mathrm{cm}^{2}\right)$ were normalized by the square of the height $\left(\mathrm{m}^{2}\right)$ to obtain the normalized SMI. The handgrip strength was measured using a digital hand dynamometer (TKK 5401 Grip-D; Takei, Tokyo, Japan) with a precision of $0.1 \mathrm{~kg}$. With the patient standing upright, we measured the left and right grip strength twice, and adopted the higher average.

\section{Evaluation of PA}

PA was evaluated using the International Physical Activity Questionnaire (usual 7 days, short version) (IPAQ) [28-30], which comprises four questions: vigorous intensity, moderate intensity, walking, sitting and lying down time. PA (metabolic equivalents (METs)-h/week) was estimated by adding the products of reported time for each item by the METs value that was specific to each PA category.

\section{Statistical analysis}

Statistical analyses were performed using EZR ver. 1.37 (Saitama Medical Center, Jichi Medical University, Saitama, Japan) [31]. Continuous variables were represented by median (interquartile range) or mean (standard deviation) and compared using the Mann-Whitney $U$ test or Student's $t$-test. Categorical variables and nominal variables were expressed in frequency (percentage) and compared by Fisher's exact test. Continuous variables showing a significant association with sarcopenia in univariate analyses were entered into a multivariable logistic regression model, after cut-off values were calculated by re- 
Table 1. Comparison of the Two Groups With and Without Sarcopenia

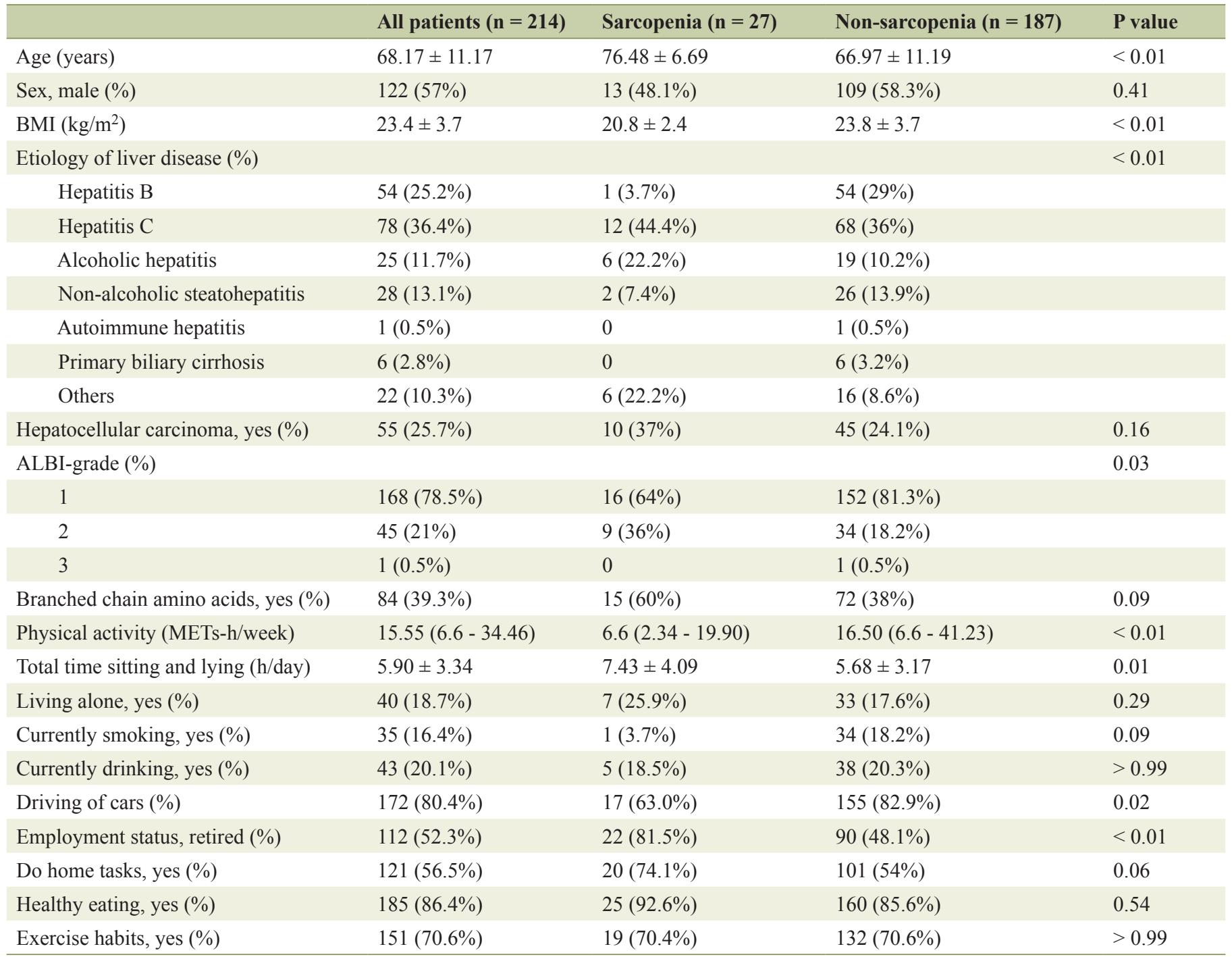

Data were expressed as means \pm standard deviation or as median (interquartile range) or as $\mathrm{n}(\%)$. $\mathrm{P}<0.05$ was considered significant. Categorical variables were compared by Fisher's exact test and continuous variables were compared by Student's $t$-tests or Mann-Whitney $U$ test. BMI, body mass index.

ceiver-operating characteristic curve (ROC) analysis. Values of $P<0.05$ were considered statistically significant.

\section{Results}

\section{Characteristics of sarcopenia in patients with chronic liver disease}

A total of 214 patients were enrolled into the present study. Of those, $27(12.6 \%)$ patients were diagnosed with sarcopenia. The patients were divided into two groups based on the presence or absence of sarcopenia, and we compared patient characteristics, PA, and the total time sitting and lying during a single day between the groups (Table 1). Sarcopenia patients were older than non-sarcopenia patients $(76.48 \pm 6.69$ versus 66.97 \pm 11.19 years old; $\mathrm{P}<0.01)$, and had lower BMIs $(20.84 \pm 2.44$ versus $\left.23.76 \pm 3.72 \mathrm{~kg} / \mathrm{m}^{2} ; \mathrm{P}<0.01\right)$, lower PA (6.6 (2.34 19.90) versus 16.5 (6.60 - 41.23) METs-h/week; $\mathrm{P}<0.01)$ and longer total time sitting and lying times per day $(7.43 \pm 4.09$ versus $5.68 \pm 3.17 \mathrm{~h} /$ day; $\mathrm{P}=0.01)$. Furthermore, retired status $(81.5 \%$ versus $48.1 \% ; \mathrm{P}<0.01)$ and a low frequency of driving ( $40 \%$ versus $20 \% ; \mathrm{P}=0.01)$ were more prevalent in sarcopenia versus non-sarcopenia patients.

\section{Comparison of laboratory parameters}

The biochemical measurements (Table 2) revealed significantly lower values of serum Alb, CK and ChE, and significantly higher values for the CONUT-score and Fib4-index in sarco- 
Table 2. Comparison of Biochemical Measurements

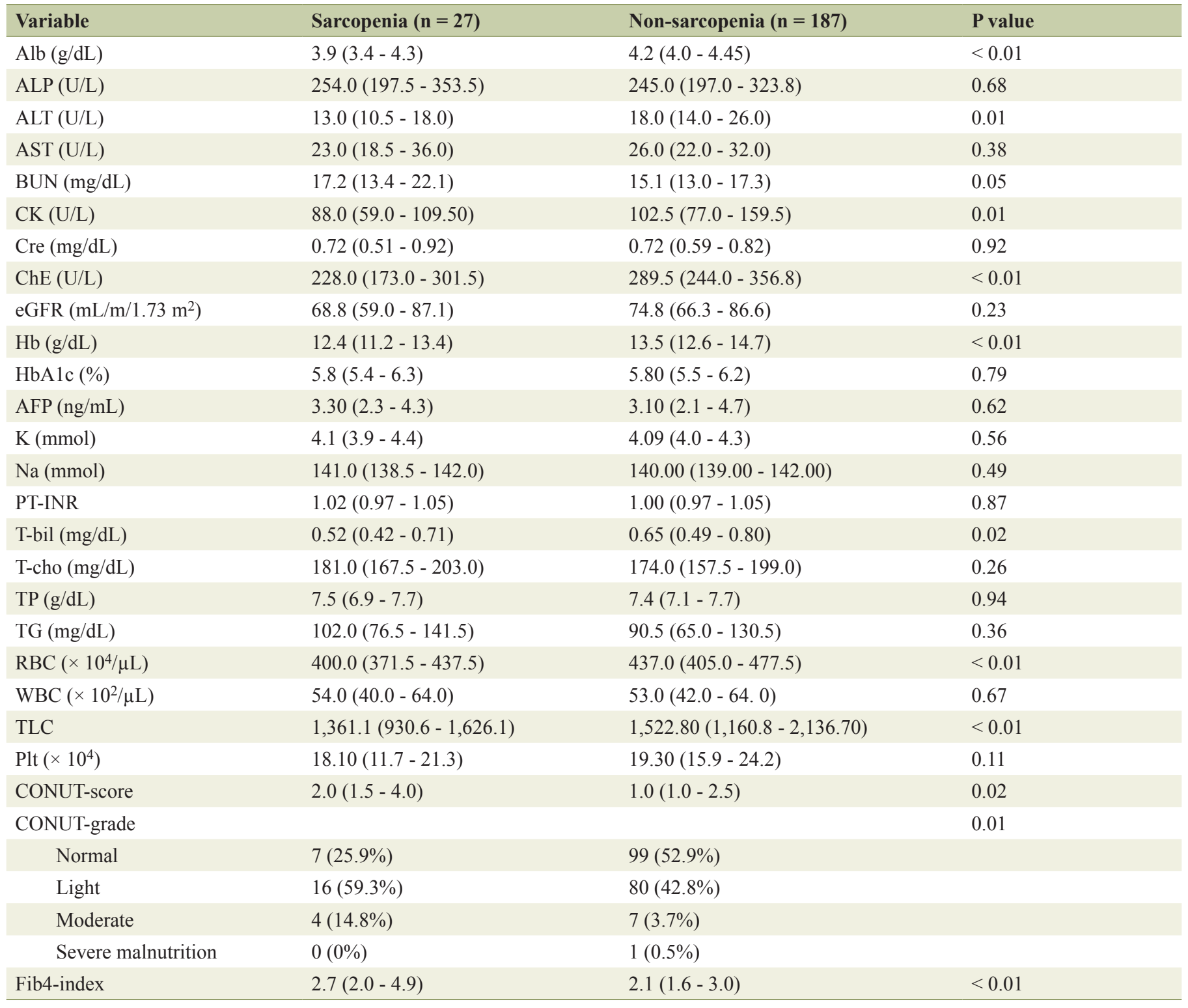

Data were expressed as median (interquartile range) or as $\mathrm{n}(\%) . \mathrm{P}<0.05$ was considered significant. Categorical variables were compared by Fisher's exact test and continuous variables were compared by Mann-Whitney $U$ test. Alb: serum albumin; ALP: alkaline phosphatase; ALT: alanine transaminase; AST: aspartate aminotransferase; BUN: blood urea nitrogen; CK: creatine kinase; Cre: creatinine; ChE: cholinesterase; eGFR: estimated glomerular filtration rate; Hb: hemoglobin; HbA1c: hemogobinA1c; AFP: $\alpha$-fetoprotein; K: potassium; Na: sodium; PT-INR: prothrombin timeinternational normalized ratio; T-bil: total bilirubin; T-cho: total cholesterol; TP: total protein; TG: triglyceride; RBC: red blood cell count; WBC: white blood cell count; TLC: total lymphocyte count; PIt: platelet count; CONUT-score: controlling nutritional status index score; Fib4-index: fibrosis 4 index.

penia patients than in non-sarcopenia patients.

\section{Predictive factors of sarcopenia in patients with chronic liver disease}

The cut-off values of age, BMI, PA, and the total time sitting and lying on the day were calculated by ROC. The cut-off value of age was chosen at 71.0 years, with an area under the curve (AUC) of 0.772 (95\% confidence interval (CI): 0.688 -
$0.855, \mathrm{P}<0.001)$, while the cut-off value of BMI was $22.2 \mathrm{~kg} /$ $\mathrm{m}^{2}$, with an AUC of 0.736 (95\% CI: $\left.0.655-0.817, \mathrm{P}<0.001\right)$. The cut-off value of PA was chosen at $10.2 \mathrm{METs}-\mathrm{h} /$ week, with an AUC of 0.674 (95\% CI: $0.570-0.779, \mathrm{P}=0.003)$. The cut-off value of the total time sitting and lying on the day was $8.0 \mathrm{~h} /$ day, with an AUC of 0.637 (95\% CI: $0.511-0.763, \mathrm{P}=$ 0.021] (Fig. 1). Logistic regression analyses identified the independent predictive factors for sarcopenia as age (odds ratio (OR): 5.89, 95\% CI: 2.15 - 16.20; P < 0.01), BMI (OR: 4.77, 95\% CI: $1.87-12.10 ; \mathrm{P}<0.01)$ and PA (OR: 3.65, 95\% CI: 
a)

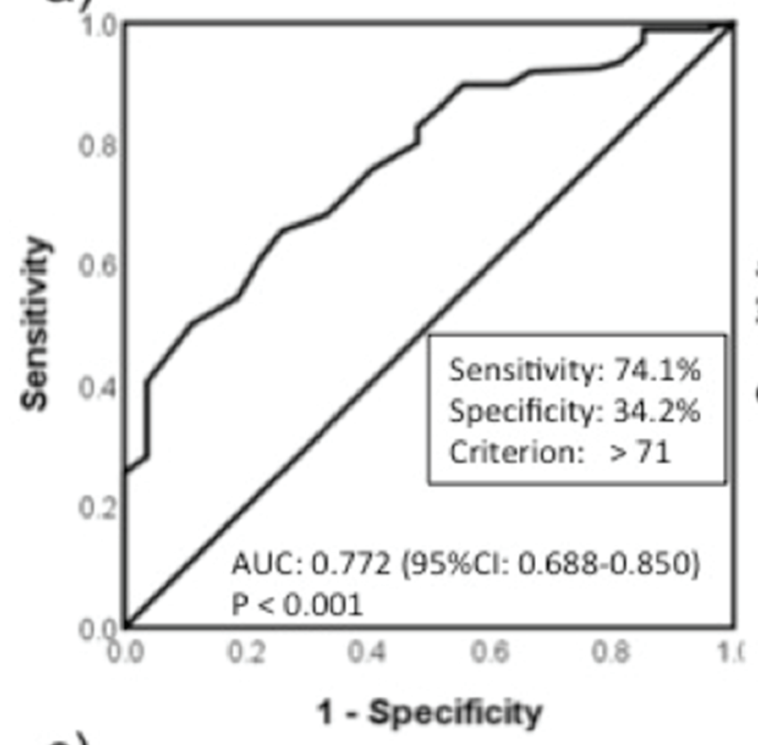

c)

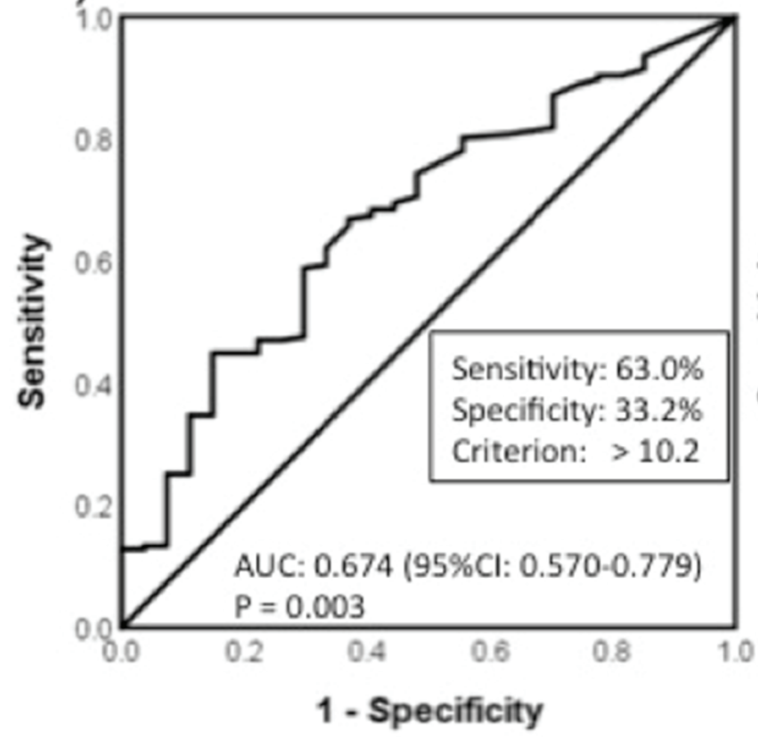

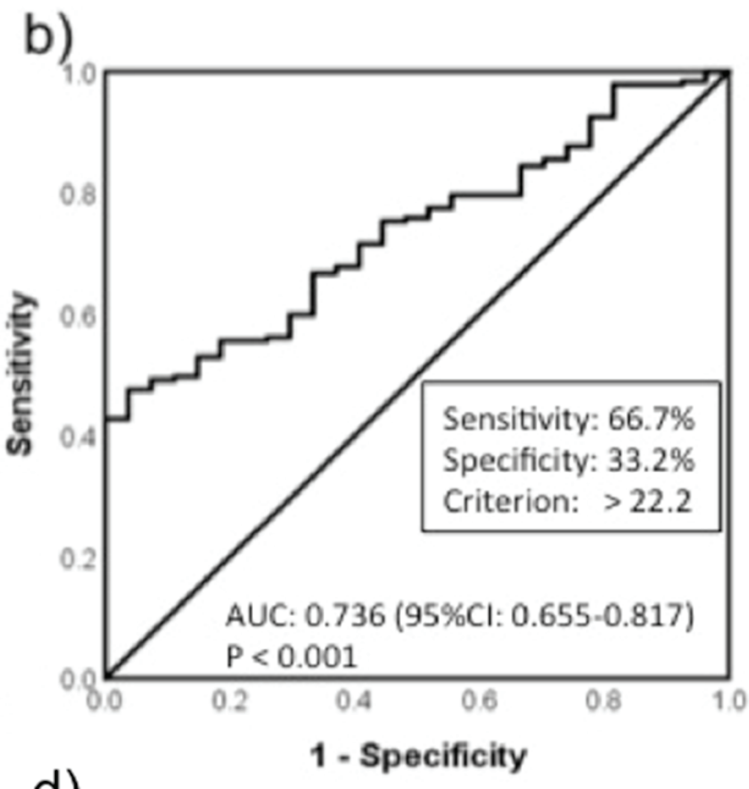

d)

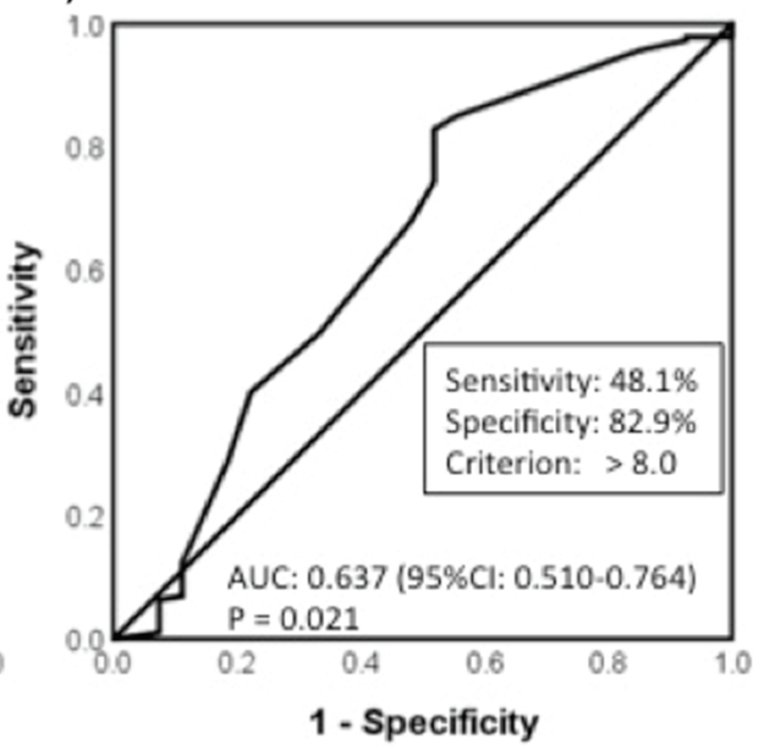

Figure 1. ROC analysis. (a) age; (b) body mass index; (c) physical activity; (d) total time of sitting and lying on the day. AUC: area under the curve; $\mathrm{Cl}$ : confidence interval; $\mathrm{BMI}$ : body mass index.

Table 3. Logistic Regression Analysis of Variables Associated With Sarcopenia

\begin{tabular}{|c|c|c|c|c|c|c|}
\hline \multirow{2}{*}{ Variable } & \multicolumn{3}{|c|}{ Univariate analysis } & \multicolumn{3}{|c|}{ Multivariate analysis } \\
\hline & OR & $95 \% \mathrm{CI}$ & P value & OR & $95 \% \mathrm{CI}$ & P value \\
\hline Age $(\geq 71 /<71$ years $)$ & 5.42 & $2.00-17.22$ & $<0.01$ & 5.89 & $2.15-16.20$ & $<0.01$ \\
\hline BMI $(<22.2 / \geq 22.2)$ & 3.91 & $1.56-1048$ & $<0.01$ & 4.77 & $1.87-12.10$ & $<0.01$ \\
\hline Low frequency of driving (yes/no) & 2.83 & $1.06-7.28$ & 0.02 & & & \\
\hline
\end{tabular}

Data were analyzed by logistic regression analysis model. OR: odds ratio; Cl: confidence interval; BMI: body mass index. 


\subsection{5 - 16.20; $\mathrm{P}<0.01$ ) (Table 3).}

\section{Discussion}

\section{Frequency of sarcopenia}

The prevalence of sarcopenia among patient with chronic liver disease in this study was $12.6 \%$, which is within reported values of $11-68 \%$ from previous studies in Japan [16]. The diagnostic criteria for sarcopenia are based on SMI evaluated by CT; however, the cut-off value of SMI is not constant, and the prevalence of sarcopenia differs widely among reports. For example, the prevalence of sarcopenia was $26.7 \%$ [32] or $11.6 \%$ [33], according to studies that diagnosed sarcopenia using the same criteria as in this study. According to Hanai et al [32], $73 \%$ of patients with HCC were included, possibly accounting for the higher prevalence compared to this study. On the other hand, a report by Hayashi et al [33] is close to this study that prevalence of sarcopenia was $12.3 \%$, with a similar patient population studied (median of 65 years old), excluding patients with the presence of active malignancy. Therefore, the population studied herein is presumed to represent the general group of chronic liver disease patients in Japan.

\section{Evaluation of PA}

The PA of the sarcopenia patients in this study was 6.6 METs-h/ week (median). In addition, the total time sitting and lying on the day was longer in the sarcopenia group than that for nonsarcopenia patients. The World Health Organization (WHO) recommends that older adults should do at least $150 \mathrm{~min}$ of moderate-intensity aerobic PA throughout the week or at least 75 min of vigorous-intensity aerobic PA throughout the week or an equivalent combination of moderate- and vigorous-intensity activity to achieve these health benefits [34]. The 150 min of moderate and $75 \mathrm{~min}$ of vigorous activity correspond to $600 \mathrm{METs}-\mathrm{min} / \mathrm{week}$, which is close to the cut-off value of PA (10.2 METs-h/week = 612 METs-min/week) in this study. Thus, PA of 600 METs-min/week could be effective for preventing sarcopenia in chronic liver disease patients over 65 years of age. Furthermore, moderate exercise in patients with cirrhosis increased muscle mass and improved physical function [18]. On the other hand, longer sedentary time decreases the physical function of the elderly [35]. Therefore, both reducing sedentary time and promoting PA during the daily life of liver disease patients may be useful for preventing sarcopenia.

Sarcopenia patients may spend decreased time outside, supported by our results showing lower driving frequency in the sarcopenia patients compared with the non-sarcopenia patients. People in the suburbs with less public transport often go out by car, and for this group, a reduction in driving frequency could further reduce out-of-home activity [36]. Ultimately, poor transportation reduces going out. Then, it may be caused the decrease in PA and the increase in sedentary time at home.

\section{Relationship between malnutrition or liver fibrosis and sarcopenia}

Sarcopenia patients in this study showed a higher CONUTscore and Fib4-index than non-sarcopenia patients. A higher CONUT-score indicates increased malnutrition [23], while a higher Fib4-index means more advanced liver fibrosis [26]. Malnutrition is one of the most frequent complications in cirrhotic patients [37], and a cause of sarcopenia [2]. The current treatment strategy for sarcopenia is administration of branched chain amino acids (BCAA) and increased protein intake $[8,38]$; however, $40 \%$ of the sarcopenia patients in this study had not received BCAA. Indeed, in Japan it is highly probable that $\mathrm{BCAA}$ have not been administered to patients with sarcopenia with Alb $>3.5 \mathrm{~g} / \mathrm{dL}$, even in the presence of liver disease. In addition, it has been reported that physical exercise therapy combined with BCAA can improve sarcopenia $[39,40]$. Therefore, assessing dietary intake and nutritional status and increasing PA could help to prevent sarcopenia in daily life.

\section{Limitations}

There are several limitations in the present study. First, the definition of sarcopenia in this study is different from that stated by the EWGSOP and Asia Working Group for Sarcopenia (AWGS) $[2,4]$; in particular, it does not distinguish between age and gait speed, leading to a potential difference in diagnostic criteria. Second, because PA was measured by questionnaire in the present study, the accuracy of the analysis could be limited and we believe that future evaluations should use an accelerometer. Finally, this was a cross-sectional study, thus, we could not absolutely verify a causal relationship between PA or lifestyle and sarcopenia in patients with chronic liver disease. Future cohort studies are therefore needed.

\section{Conclusions}

Patients with chronic liver disease in this study had a prevalence of sarcopenia of $12.6 \%$. Sarcopenia patients' lifestyle characteristics were longer sedentary time, lower frequency of driving and higher rate of retirement. Independent predictive factors of sarcopenia were advanced age, low BMI and low PA. For these patients, lifestyle interventions to prevent sarcopenia may be effective.

\section{Conflict of Interest}

All authors have no conflict of interest to declare.

\section{Financial Support}

This work was supported financially by Niigata University Graduate School of Health Sciences. 


\section{References}

1. Rosenberg I. Summary comments: epidemiological and methodological problems in determining nutritional status of older persons. Am J Clin Nutr. 1989;50:1231-1233.

2. Cruz-Jentoft AJ, Baeyens JP, Bauer JM, Boirie Y, Cederholm T, Landi F, Martin FC, et al. Sarcopenia: European consensus on definition and diagnosis: Report of the European Working Group on Sarcopenia in Older People. Age Ageing. 2010;39(4):412-423.

3. Goodpaster BH, Park SW, Harris TB, Kritchevsky SB, Nevitt M, Schwartz AV, Simonsick EM, et al. The loss of skeletal muscle strength, mass, and quality in older adults: the health, aging and body composition study. J Gerontol A Biol Sci Med Sci. 2006;61(10):1059-1064.

4. Chen LK, Liu LK, Woo J, Assantachai P, Auyeung TW, Bahyah KS, Chou MY, et al. Sarcopenia in Asia: consensus report of the Asian Working Group for Sarcopenia. J Am Med Dir Assoc. 2014;15(2):95-101.

5. Evans WJ. Skeletal muscle loss: cachexia, sarcopenia, and inactivity. Am J Clin Nutr. 2010;91(4):1123S-1127S.

6. Buford TW, Anton SD, Judge AR, Marzetti E, Wohlgemuth SE, Carter CS, Leeuwenburgh C, et al. Models of accelerated sarcopenia: critical pieces for solving the puzzle of age-related muscle atrophy. Ageing Res Rev. 2010;9(4):369-383.

7. Papa EV, Dong X, Hassan M. Resistance training for activity limitations in older adults with skeletal muscle function deficits: a systematic review. Clin Interv Aging. 2017;12:955-961.

8. Dasarathy S. Consilience in sarcopenia of cirrhosis. J Cachexia Sarcopenia Muscle. 2012;3(4):225-237.

9. Fujiwara N, Nakagawa H, Kudo Y, Tateishi R, Taguri M, Watadani T, Nakagomi R, et al. Sarcopenia, intramuscular fat deposition, and visceral adiposity independently predict the outcomes of hepatocellular carcinoma. J Hepatol. 2015;63(1):131-140.

10. Kamachi S, Mizuta T, Otsuka T, Nakashita S, Ide Y, Miyoshi A, Kitahara K, et al. Sarcopenia is a risk factor for the recurrence of hepatocellular carcinoma after curative treatment. Hepatol Res. 2016;46(2):201-208.

11. Itoh S, Shirabe K, Matsumoto Y, Yoshiya S, Muto J, Harimoto N, Yamashita Y, et al. Effect of body composition on outcomes after hepatic resection for hepatocellular carcinoma. Ann Surg Oncol. 2014;21(9):3063-3068.

12. Harimoto N, Shirabe K, Yamashita YI, Ikegami T, Yoshizumi T, Soejima Y, Ikeda T, et al. Sarcopenia as a predictor of prognosis in patients following hepatectomy for hepatocellular carcinoma. Br J Surg. 2013;100(11):15231530.

13. Masuda T, Shirabe K, Ikegami T, Harimoto N, Yoshizumi T, Soejima Y, Uchiyama H, et al. Sarcopenia is a prognostic factor in living donor liver transplantation. Liver Transpl. 2014;20(4):401-407.

14. Iritani $\mathrm{S}$, Imai $\mathrm{K}$, Takai $\mathrm{K}$, Hanai $\mathrm{T}$, Ideta T, Miyazaki $\mathrm{T}$, Suetsugu A, et al. Skeletal muscle depletion is an independent prognostic factor for hepatocellular carcinoma. J Gastroenterol. 2015;50(3):323-332.
15. Montano-Loza AJ. Clinical relevance of sarcopenia in patients with cirrhosis. World J Gastroenterol. 2014;20(25):8061-8071.

16. Nishikawa H, Shiraki M, Hiramatsu A, Moriya K, Hino K, Nishiguchi S. Japan Society of Hepatology guidelines for sarcopenia in liver disease (1st edition): Recommendation from the working group for creation of sarcopenia assessment criteria. Hepatol Res. 2016;46(10):951-963.

17. Zenith L, Meena N, Ramadi A, Yavari M, Harvey A, Carbonneau M, Ma M, et al. Eight weeks of exercise training increases aerobic capacity and muscle mass and reduces fatigue in patients with cirrhosis. Clin Gastroenterol Hepatol. 2014;12(11):1920-1926 e11922.

18. Roman E, Garcia-Galceran C, Torrades T, Herrera S, Marin A, Donate M, Alvarado-Tapias E, et al. Effects of an exercise programme on functional capacity, body composition and risk of falls in patients with cirrhosis: a randomized clinical trial. PLoS One. 2016;11(3):e0151652.

19. Zhou P, Hughes AK, Grady SC, Fang L. Physical activity and chronic diseases among older people in a mid-size city in China: a longitudinal investigation of bipolar effects. BMC Public Health. 2018;18(1):486.

20. Dunn MA, Josbeno DA, Schmotzer AR, Tevar AD, DiMartini AF, Landsittel DP, Delitto A. The gap between clinically assessed physical performance and objective physical activity in liver transplant candidates. Liver Transpl. 2016;22(10):1324-1332.

21. Salo J, Guevara M, Fernandez-Esparrach G, Bataller R, Gines A, Jimenez W, Gines P, et al. Impairment of renal function during moderate physical exercise in cirrhotic patients with ascites: relationship with the activity of neurohormonal systems. Hepatology. 1997;25(6):1338-1342.

22. Trivedi HD, Tapper EB. Interventions to improve physical function and prevent adverse events in cirrhosis. Gastroenterol Rep (Oxf). 2018;6(1):13-20.

23. Ignacio de Ulibarri J, Gonzalez-Madrono A, de Villar NG, Gonzalez P, Gonzalez B, Mancha A, Rodriguez F, et al. CONUT: a tool for controlling nutritional status. First validation in a hospital population. Nutr Hosp. 2005;20(1):38-45.

24. Vallet-Pichard A, Mallet V, Nalpas B, Verkarre V, Nalpas A, Dhalluin-Venier V, Fontaine H, et al. FIB-4: an inexpensive and accurate marker of fibrosis in $\mathrm{HCV}$ infection. comparison with liver biopsy and fibrotest. Hepatology. 2007;46(1):32-36.

25. Sumida Y, Yoneda M, Hyogo H, Itoh Y, Ono M, Fujii H, Eguchi Y, et al. Validation of the FIB4 index in a Japanese nonalcoholic fatty liver disease population. BMC Gastroenterol. 2012;12:2.

26. Sterling RK, Lissen E, Clumeck N, Sola R, Correa MC, Montaner J, M SS, et al. Development of a simple noninvasive index to predict significant fibrosis in patients with HIV/HCV coinfection. Hepatology. 2006;43(6):13171325.

27. Johnson PJ, Berhane S, Kagebayashi C, Satomura S, Teng M, Reeves HL, O'Beirne J, et al. Assessment of liver function in patients with hepatocellular carcinoma: a new evidence-based approach-the ALBI grade. J Clin Oncol. 2015;33(6):550-558. 
28. Craig CL, Marshall AL, Sjostrom M, Bauman AE, Booth ML, Ainsworth BE, Pratt M, et al. International physical activity questionnaire: 12 -country reliability and validity. Med Sci Sports Exerc. 2003;35(8):1381-1395.

29. Tomioka K, Iwamoto J, Saeki K, Okamoto N. Reliability and validity of the International Physical Activity Questionnaire (IPAQ) in elderly adults: the Fujiwara-kyo Study. J Epidemiol. 2011;21(6):459-465.

30. Murase N, Katsumura T, Ueda C, Inoue S, Shimomitsu T. Validity and reliability of Japanese version of international physical activity questionnaire. J Heal Welf Stat. 2002;49:1-9.

31. Kanda Y. Investigation of the freely available easy-touse software 'EZR' for medical statistics. Bone Marrow Transplant. 2013;48(3):452-458.

32. Hanai T, Shiraki M, Watanabe S, Kochi T, Imai K, Suetsugu A, Takai K, et al. Sarcopenia predicts minimal hepatic encephalopathy in patients with liver cirrhosis. Hepatol Res. 2017;47(13):1359-1367.

33. Hayashi M, Abe K, Fujita M, Okai K, Takahashi A, Ohira H. Association between sarcopenia and osteoporosis in chronic liver disease. Hepatol Res. 2018;48(11):893-904.

34. World Health Organization (WHO): Global recommendations on physical activity for health. 2010. http://apps. who.int/iris/bitstream/10665/44399/1/9789241599979 eng.pdf. Accessed 28 May 2018.
35. Rosenberg DE, Bellettiere J, Gardiner PA, Villarreal VN, Crist K, Kerr J. Independent associations between sedentary behaviors and mental, cognitive, physical, and functional health among older adults in retirement communities. J Gerontol A Biol Sci Med Sci. 2016;71(1):78-83.

36. Marottoli RA, de Leon CFM, Glass TA, Williams CS, Cooney LM, Jr., Berkman LF. Consequences of driving cessation: decreased out-of-home activity levels. J Gerontol B Psychol Sci Soc Sci. 2000;55(6):S334-340.

37. Lochs H, Plauth M. Liver cirrhosis: rationale and modalities for nutritional support - the European Society of Parenteral and Enteral Nutrition consensus and beyond. Curr Opin Clin Nutr Metab Care. 1999;2(4):345-349.

38. Landi F, Calvani R, Tosato M, Martone AM, Ortolani E, Savera G, D'Angelo E, et al. Protein intake and muscle health in old age: from biological plausibility to clinical evidence. Nutrients. 2016;8(5):295.

39. Kim HK, Suzuki T, Saito K, Yoshida H, Kobayashi H, Kato H, Katayama M. Effects of exercise and amino acid supplementation on body composition and physical function in community-dwelling elderly Japanese sarcopenic women: a randomized controlled trial. J Am Geriatr Soc. 2012;60(1):16-23.

40. Toshikuni N, Arisawa T, Tsutsumi M. Nutrition and exercise in the management of liver cirrhosis. World J Gastroenterol. 2014;20(23):7286-7297. 